\title{
Article \\ A Shortcut from Metabolic-Associated Fatty Liver Disease (MAFLD) to Hepatocellular Carcinoma (HCC): c-MYC a Promising Target for Preventative Strategies and Individualized Therapy
}

Feifei Guo 1,2,+, Olga Estévez-Vázquez ${ }^{1,+}{ }^{-}$, Raquel Benedé-Ubieto ${ }^{1,3}$, Douglas Maya-Miles ${ }^{4,5,6}$, Kang Zheng 1,7, Rocío Gallego-Durán 4,5,6, Ángela Rojas 4,5,6, Javier Ampuero 4,5,6, Manuel Romero-Gómez 4,5,6,8 (D, Kaye Philip 9 , Isioma U. Egbuniwe ${ }^{9}\left(\mathbb{D}\right.$, Chaobo Chen ${ }^{1,10,11}$, Jorge Simon ${ }^{6,12} \mathbb{D}$, Teresa C. Delgado ${ }^{12}(\mathbb{D}$, María Luz Martínez-Chantar 6,12 ${ }^{\mathbb{D}}$, Jie Sun ${ }^{7}$, Johanna Reissing ${ }^{13}$, Tony Bruns ${ }^{13} \mathbb{D}_{\text {, Arantza Lamas-Paz }}{ }^{1}$, Manuel Gómez del Moral ${ }^{14}$ (D), Marius Maximilian Woitok ${ }^{13}$, Javier Vaquero ${ }^{6,15,16}$, José R. Regueiro ${ }^{1}$ (D), Christian Liedtke ${ }^{13}$ (D) Christian Trautwein ${ }^{13}$, Rafael Bañares ${ }^{1,6,15,16}$, Francisco Javier Cubero $1,6,16, \neq(\mathbb{D})$ and Yulia A. Nevzorova $1,6,13,16, *, \ddagger$ (D)

check for updates

Citation: Guo, F.; Estévez-Vázquez, O.; Benedé-Ubieto, R.; Maya-Miles, D.; Zheng, K.; Gallego-Durán, R.; Rojas, Á.; Ampuero, J.; Romero-Gómez, M.; Philip, K.; et al A Shortcut from

Metabolic-Associated Fatty Liver Disease (MAFLD) to Hepatocellular Carcinoma (HCC): c-MYC a Promising Target for Preventative Strategies and Individualized Therapy. Cancers 2022, 14, 192. https://doi.org/10.3390/

cancers14010192

Academic Editor:

George Papatheodoridis

Received: 11 November 2021

Accepted: 20 December 2021

Published: 31 December 2021

Publisher's Note: MDPI stays neutral with regard to jurisdictional claims in published maps and institutional affiliations.

Copyright: (C) 2021 by the authors. Licensee MDPI, Basel, Switzerland. This article is an open access article distributed under the terms and conditions of the Creative Commons Attribution (CC BY) license (https:// creativecommons.org/licenses/by/ $4.0 /)$.
1 Department of Immunology, Ophthalmology and ENT, School of Medicine, Complutense University of Madrid, 12 de Octubre (imas12) Health Research Institute, 28040 Madrid, Spain; feiguo@ucm.es (F.G.); olgaeste@ucm.es (O.E.-V.); rabenede@ucm.es (R.B.-U.); kzheng@ucm.es (K.Z.); chaochen@ucm.es (C.C.); arlamas@ucm.es (A.L.-P.); regueiro@med.ucm.es (J.R.R.); Rafael.banares@salud.madrid.org (R.B.); fcubero@ucm.es (F.J.C.)

2 Department of Obstetrics and Gynaecology, The Affiliated Drum Tower Hospital of Nanjing University Medical School, Nanjing 210023, China

3 Department of Physiology, Genetics and Microbiology, Faculty of Biology, Complutense University Madrid, 28040 Madrid, Spain

4 Institute of Biomedicine of Seville (IBiS), SeLiver Group, Virgen del Rocío University Hospital/CSIC/University of Seville, 41013 Seville, Spain; dmaya-ibis@us.es (D.M.-M.); rgallego-ibis@us.es (R.G.-D.); marojas-ibis@us.es (Á.R.); jampuero-ibis@us.es (J.A.); mromerogomez@us.es (M.R.-G.)

5 UCM Digestive Diseases, Virgen del Rocío University Hospital, 41013 Seville, Spain

6 Centro de Investigación Biomédica en Red de Enfermedades Hepáticas y Digestivas (CIBEREHD), 28220 Madrid, Spain; jsimon.ciberehd@cicbiogune.es (J.S.); mlmartinez@cicbiogune.es (M.L.M.-C.) j.vaquero@iisgm.com (J.V.)

7 Department of Anesthesiology, Zhongda Hospital, School of Medicine, Southeast University, Nanjing 210009, China; sunjie@seu.edu.cn

8 Department of Medicine, University of Seville, 41009 Seville, Spain

9 Department of Pathology, Nottingham University Hospitals NHS Trust, Queen's Medical Centre Campus, Nottingham NG7 2UH, UK; Philip.Kaye@nuh.nhs.uk (K.P.); Isioma.Egbuniwe@nottingham.ac.uk (I.U.E.)

10 Department of General Surgery, Wuxi Xishan People's Hospital, Wuxi 214000, China

11 Department of Hepatic-Biliary-Pancreatic Surgery, The Affiliated Drum Tower Hospital of Nanjing University Medical School, Nanjing 210023, China

12 Liver Disease Laboratory, Center for Cooperative Research in Biosciences (CIC bioGUNE), Basque Research and Technology Alliance (BRTA), 48160 Derio, Spain; tcardoso@cicbiogune.es

13 Department of Internal Medicine III, University Hospital RWTH Aachen, 52074 Aachen, Germany; joreissing@ukaachen.de (J.R.); tbruns@ukaachen.de (T.B.); M.Woitok@eclevar.com (M.M.W.); cliedtke@ukaachen.de (C.L.); ctrautwein@ukaachen.de (C.T.)

14 Department of Cell Biology, Complutense University School of Medicine, 28040 Madrid, Spain; mgomezm@med.ucm.es

15 Servicio de Aparato Digestivo, Hospital General Universitario Gregorio Marañón, 28009 Madrid, Spain

16 Instituto de Investigación Sanitaria Gregorio Marañón (IiSGM), 28007 Madrid, Spain

* Correspondence: yulianev@ucm.es; Tel.: +49-(0)241-80-80662; Fax: +49-(0)241-80-82455

+ These authors contributed equally to this work.

$\ddagger$ These authors contributed equally to this work.

Simple Summary: Metabolic-associated fatty liver disease (MAFLD) is a chronic liver disease associated with obesity, diabetes mellitus type 2 (DM2), and hyperlipidemia. It can also progress to end-stage hepatocellular carcinoma (HCC); the underlying mechanisms are still unknown, but endogenous (i.e., genetic) factors such as oncogenes have been suggested to play a role. We found 
that c-MYC transgenic mice with ageing are prone to develop obesity, metabolic syndrome (MS), and abnormal accumulation of lipids in the liver compared to control mice. A short-term application of the Western diet (WD) significantly worsened the phenotype and accelerate HCC development. Importantly, we found that metformin as therapeutic approach significantly attenuated MAFLD phenotype in transgenic mice. We also observed that c-MYC is up-regulated in human patients with MAFLD and MAFLD-related HCC. Altogether the current study suggests an important role of the oncogene c-MYC during the progression from MAFLD to HCC and makes c-MYC a possible target for preventative strategies and individualized therapy.

Abstract: Background: Metabolic-associated fatty liver disease (MAFLD) has risen as one of the leading etiologies for hepatocellular carcinoma (HCC). Oncogenes have been suggested to be responsible for the high risk of MAFLD-related HCC. We analyzed the impact of the proto-oncogene c-MYC in the development of human and murine MAFLD and MAFLD-associated HCC. Methods: alb-myctg mice were studied at baseline conditions and after administration of Western diet (WD) in comparison to WT littermates. c-MYC expression was analyzed in biopsies of patients with MAFLD and MAFLD-associated HCC by immunohistochemistry. Results: Mild obesity, spontaneous hyperlipidaemia, glucose intolerance and insulin resistance were characteristic of 36-week-old alb-myc ${ }^{\text {tg }}$ mice. Middle-aged alb-myc ${ }^{\mathrm{tg}}$ exhibited liver steatosis and increased triglyceride content. Liver injury and inflammation were associated with elevated ALT, an upregulation of ER-stress response and increased ROS production, collagen deposition and compensatory proliferation. At 52 weeks, $20 \%$ of transgenic mice developed HCC. WD feeding exacerbated metabolic abnormalities, steatohepatitis, fibrogenesis and tumor prevalence. Therapeutic use of metformin partly attenuated the spontaneous MAFLD phenotype of alb-myctg mice. Importantly, upregulation and nuclear localization of c-MYC were characteristic of patients with MAFLD and MAFLD-related HCC. Conclusions: A novel function of c-MYC in MAFLD progression was identified opening new avenues for preventative strategies.

Keywords: metabolic-associated fatty liver disease (MAFLD); c-myc; oncogene; tumorigenesis; metformin

\section{Introduction}

Metabolic dysfunction-associated fatty liver disease (MAFLD) is a spectrum of chronic liver diseases characterized by excessive accumulation of triglycerides (TG) in hepatocytes. The condition ranges from isolated excessive hepatocyte TG accumulation and steatosis (fatty liver (FL)), to hepatic TG accumulation plus inflammation and hepatocyte injury (steatohepatitis $(\mathrm{SH})$ ) and finally to hepatic fibrosis and cirrhosis and/or end-stage hepatocellular carcinoma (HCC) [1].

Since its first description in the early 1980s, as "a poorly understood and hitherto unnamed liver disease", MAFLD has considerably evolved and progressively gained recognition among clinical and research hepatologists [2,3]. Forty years later, the alarming epidemic of obesity and diabetes mellitus type 2 (DM2), has fueled an increasing prevalence of MAFLD and rendered it a societal health problem worldwide with a prevalence up to $24 \%$ worldwide. For example, by 2015, MAFLD cases in the US were estimated to affect 83.1 million people and predicted to increase over 100 million by 2030 [4]. In Spain, the total prevalence of MAFLD was estimated in 10.53 million cases in 2016 with a further potential to increase to 12.7 million by $2030[1,5]$.

Patients with MAFLD commonly consume high amounts of processed food with high fructose levels and/or high fat content and tend to adopt a sedentary lifestyle with low levels of physical activity. However, besides these environmental or exogenous factors many other factors often determine the progression of MAFLD and the development of MAFLD-associated carcinogenesis. For instance, $42 \%$ of MAFLD patients develop advanced $\mathrm{SH}$ stages and only $2.4-12.4 \%$ finally develop HCC [6]. Altogether, the huge variation 
in the susceptibility to the disease progression clearly indicates that among risk factors, endogenous (i.e., genetic) factors are of major relevance.

The identification of such risk factors, which synergistically with environmental factors (such as unhealthy eating habits) drive MAFLD in the direction of cancer development would have great benefits for optimization of therapeutic strategies.

The proto-oncogene c-MYC is a transcription factor which plays a central role in multiple biological processes including cell proliferation, cell growth, energy metabolism and apoptosis. Genetic alterations of c-MYC expression have been found in approximately 70,000 U.S. cancer deaths per year, including HCC [7]. Alb-myc ${ }^{\mathrm{tg}}$ mice with overexpression of c-MYC only in hepatocytes became the touchstones of experimental models of murine liver carcinogenesis due to high HCC predisposition [8].

However, the development of HCC is usually preceded by chronic liver injury and ongoing liver diseases. During the last decade, we systematically investigated the role of cMYC in chronic liver disease (CLD). We showed that upregulation of c-MYC in hepatocytes either due to gene amplification or as a response to inflammatory liver injury results in hepatic stellate cells (HSCs) preactivation. Primed HSC have a higher potential to proliferate and produce extracellular matrix (ECM) especially after a second profibrotic hit [9]. One of these hits can be an excessive alcohol consumption. We found the synergistic harmful effect of increased c-MYC expression and alcohol consumption on alcohol-associated liver disease (ALD) progression in a multistep process of exacerbated ROS production, mitochondrial and ER dysfunction, cell death, impaired cell proliferation and collagen deposition [10].

Despite the crucial recent increase in MAFLD and MAFLD-associated cancer, the specific link between c-MYC and MAFLD has not yet been addressed. In the present study, we aimed to elucidate the role of c-MYC in the initiation, progression and complications of MAFLD.

\section{Materials and Methods}

\subsection{Human Liver Samples}

Liver samples for IHC analysis were obtained from the Andalusian Biobank (Ethics Committee of the Junta de Andalucía C.P. S2100062-C.I. 0458-N-21.) from patients who were subjected to a liver biopsy for diagnostic confirmation purposes or from patients receiving a liver transplant. Diagnosis of MAFLD includes an evaluation of clinical risk factors and a histological evaluation of tissue together with the exclusion of other causes of liver disease (alcohol use disorders or excessive alcohol consumption, chronic hepatitis C or B, autoimmune, other causes). Two of these six patients did not show an obvious accumulation of fat $\geq 5 \%$ despite the presence of cirrhosis in the liver and their classification relies on clinical parameters. HCC diagnosis was performed by an experiences pathologist based on histopathological criteria (trabecular-resembling and solid patterns). Samples from HCC patients were included an IHC analysis for adjacent nontumoral and tumoral tissue. Tables S1 and S2 summarize the patients' characteristics.

\subsection{Maintenance and Treatment of Mice}

We used 12-week-old male transgenic mice carrying c-MYC transgene under the control of the hepatocyte-specific albumin promoter (alb-myc ${ }^{\mathrm{tg}}$ ) as previously described [11] and WT littermates as controls. Diets used for the development of MAFLD are shown in Tables S3 and S4.

All animal procedures were carried out according to Spanish legal requirements and animal protection law and approved by the authority of environment conservation and consumer protection of the regional government of Madrid (PROEX210/18 and PROEX125.1/20). All animals were maintained in the animal facility at the School of Biology, Complutense University of Madrid, in a temperature-controlled room with $12 \mathrm{~h}$ light/dark cycle with free access to food and water according to the guidelines of the Federation for Laboratory Animal Science Associations (FELASA). 


\subsection{Supplementary Material and Methods}

See supplementary online material for further information regarding material and methods on animal experimentation, glucose and insulin tolerance test (GTT), histological analysis, immunofluorescence (IF) and immunohistochemistry (IHC) staining, image analysis, RNA isolation and RT-qPCR, Western blot, and TG quantification.

\subsection{Statistical Analysis}

Data are expressed as mean \pm standard deviation of the mean. Statistical significance was determined by two-way analysis of variance followed by a Student's $t$ test. $p$ values for significance are indicated as follows: ${ }^{*} p<0.05 ;{ }^{* *} p<0.01$; ${ }^{* * *} p<0.001$; ${ }^{* * * *} p<0.0001$.

\section{Results}

\section{1. c-MYC Is Induced in Patients with MAFLD and MAFLD-Related HCC}

First, we analyzed the possible genetic alterations of c-MYC in a comprehensive dataset from 353 HCC patients generated by the Cancer Genome Atlas Research Network (https: / / cancergenome.nih.gov/, accessed on 16 December 2021) [12]. Genetic alterations of c-MYC, mainly amplifications, were detected in 41 patients (12\%) (Figure S1A).

Next, we narrowed down the etiology of HCC and validated the expression of c-MYC in liver biopsies from a cohort of patients with clinically and histologically diagnosed MAFLD-related HCC (Table S1). We observed a higher rate of c-MYC nuclear expression inside tumor nodules compared to corresponding tumor-adjacent areas (Figures $1 \mathrm{~A}, \mathrm{~B}$ and $\mathrm{S} 2 \mathrm{~A}$ ).

Finally, we determined the expression of c-MYC in liver samples from six patients with MAFLD and advanced liver fibrosis (F3-F4) (Table S1). c-MYC protein expression was increased and deposition was localized mainly in nuclei of hepatic cells along fibrotic areas (Figures 1C and S2B). Overall, these data demonstrated that c-MYC expression is increased in MAFLD and MAFLD-related HCC.

\subsection{Spontaneous Metabolic Syndrome Is a Feature of 36-Week-Old c-MYC Transgenic Mice}

To better define the relevance of c-MYC activation in MAFLD, we took advantage of transgenic mice with overexpression of c-MYC in hepatocytes (alb-myctg). Interestingly, alb-myc ${ }^{\text {tg }}$ mice developed rapid and significantly greater weight gain compared to control littermates on normal chow diet (Figure 2A) with similar caloric intake (Figure S3A). These changes were obvious already in 10-week-old animals, and became more pronounced as the mice grew older, and finally resulted in robust increase in the body mass index (BMI) in alb-myc ${ }^{\text {tg }}$ mice at the experimental end-point (36 weeks) (Figure 2A,B).

Accordingly, a significant increase of epididymal white adipose tissue (eWAT) in alb-myc ${ }^{\mathrm{tg}}$ mice was apparent by MRI scan images (Figure S3B) and further confirmed during necropsy (Figure 2C). Hence, H\&E staining of eWAT in alb-myctg mice revealed significant adipocyte hypertrophy and numerous crown-like structures (CLS), formed by F4-80 positive macrophages aggregates (Figures 2D and S3C).

Moreover, we found a significant increase in total cholesterol and development of hypertriglyceridemia persisting in alb-myctg from 36 weeks of age (Figure 2E). Importantly, c-MYC transgenic mice developed hyperglycaemia after $6 \mathrm{~h}$ of fasting and demonstrate considerably impaired glucose tolerance in the glucose tolerance test (GTT) (Figures 2F and S4A). These changes were accompanied by significantly delayed glucose clearance in the insulin tolerance test (ITT) 30, 60, 90 min after insulin injection (Figure S4B).

Altogether, 36-week-old alb-myc ${ }^{\text {tg }}$ mice were prone to spontaneously develop obesity, hypertriglyceridemia, hypercholesterolemia, glucose intolerance and insulin resistance (IR), characteristic of MS. 

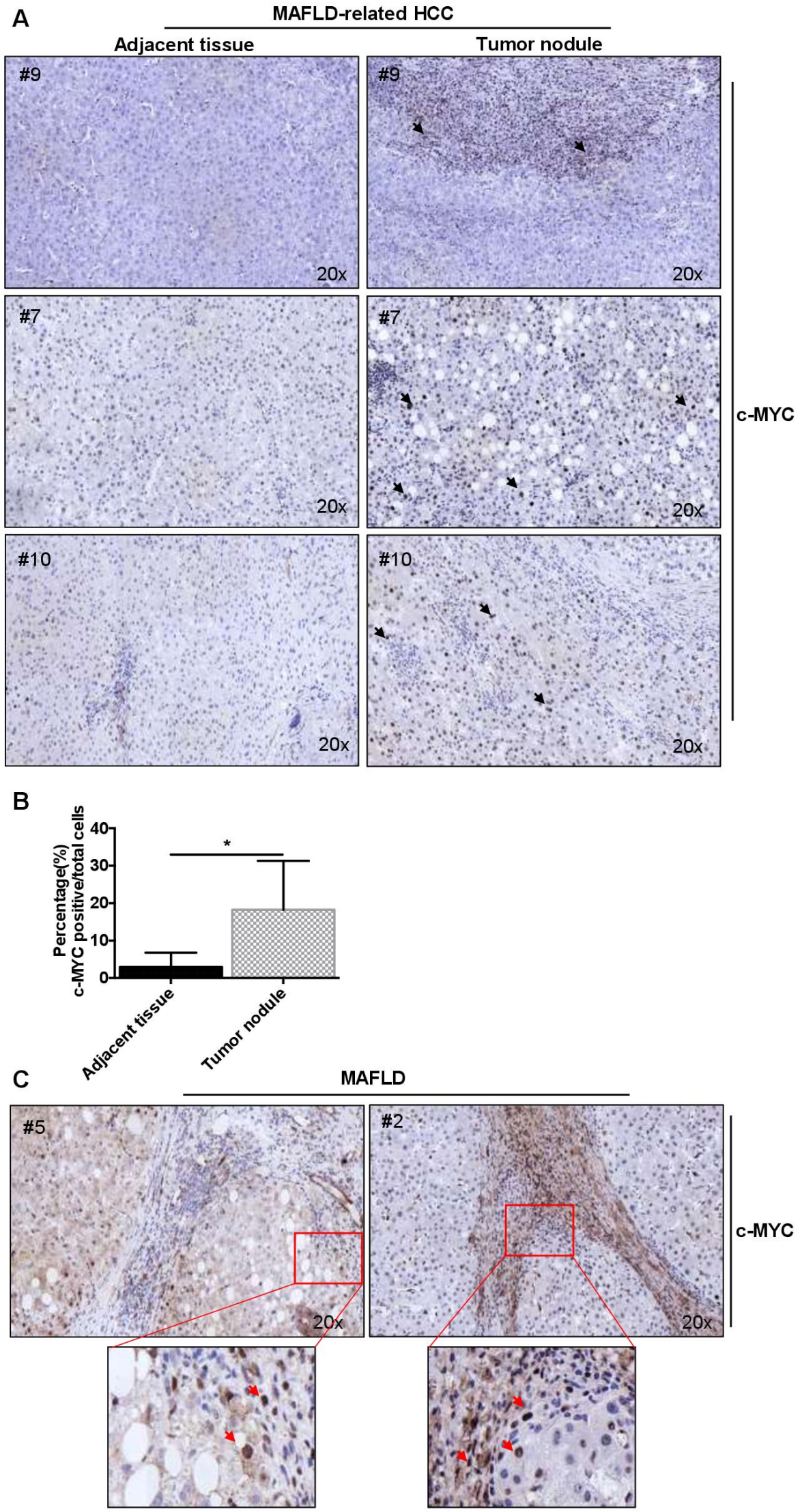

Figure 1. High expression of c-MYC in patients with MAFLD and MAFLD-related HCC. (A) c-MYC nuclear expression inside tumornodules and tumor-adjacent tissues in patients with MAFLDrelated HCC (20× magnification). Black arrows indicate cells with positive nuclear c-MYC expression 
(B) Quantification of \% c-MYC positive cells inside tumor nodules and tumor-adjacent tissues in patients with MAFLD-related HCC $(n=4)$. (C) c-MYC protein expression in MAFLD patients with advanced liver fibrosis (F3-F4), (20× magnification). Red arrows indicate cells with positive nuclear c-MYC expression. Data are expressed as the mean $\pm \mathrm{SD},{ }^{*}=p<0.05$, inside tumor nodules vs. tumor-adjacent tissues.

A

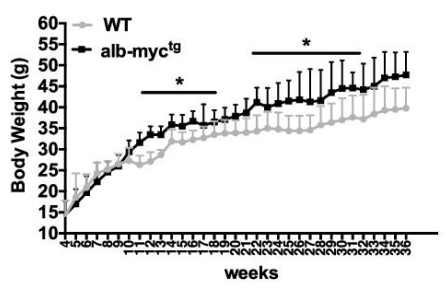

C

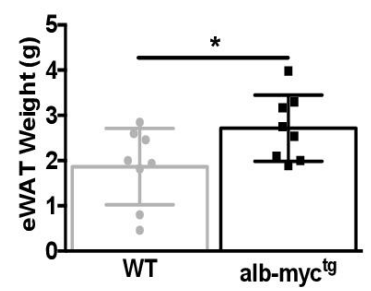

E

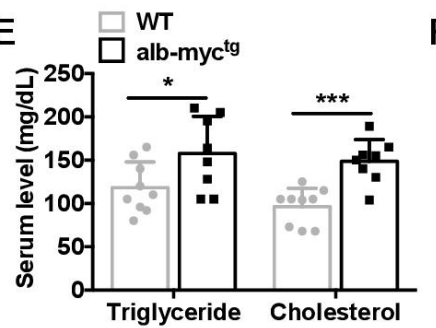

D

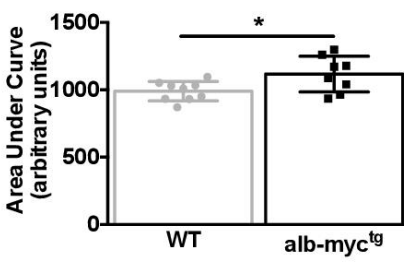

OWAT H\&E

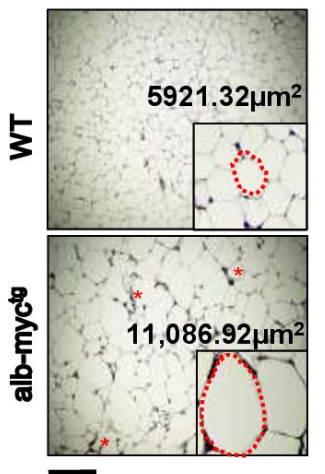

$\overline{100 \mu \mathrm{m}}$

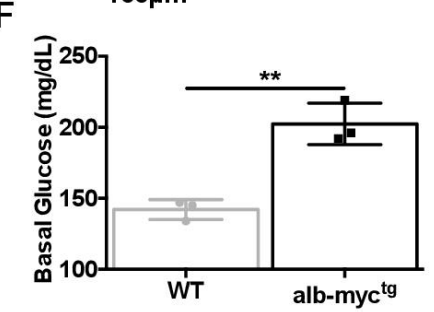

B
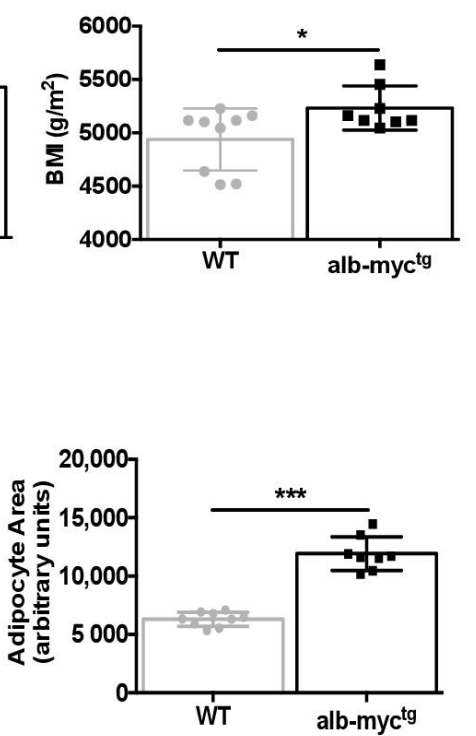

$-W T$

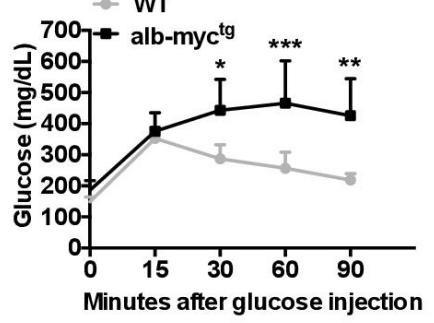

Figure 2. Metabolic profile of chow diet-fed alb-myc ${ }^{\text {tg }}$ mice at the age of 36 weeks. (A) Left: Growth curve demonstrating body weight gain, assessed at 7 days' interval for each group. Right: The histogram represents the incremental area under the respective grow curve $(n=8-9)$. (B) Body mass index (BMI) $\left(\mathrm{g} / \mathrm{m}^{2}\right)(n=8-9)$. (C) Weight of epididymal white adipose tissue (eWAT) $(n=8)$. (D) Size of eWAT cells by H\&E staining. Scale bar $=100 \mu \mathrm{m}$. Size of individual cells from both groups was measured and average cell size $\left(\mu \mathrm{m}^{2}\right)$ was quantified $(n=8-9)$. Asterisks indicate CLS. The dotted red line marks the individual adipocyte. (E) Levels of cholesterol and TG in serum $(n=8-9)$. (F) Basal glucose level in blood after $6 \mathrm{~h}$ fasting; Blood glucose concentration curve during intraperitoneal glucose tolerance test $(n=3)$. Data are expressed as the mean $\pm \mathrm{SD},{ }^{*}=p<0.05 ;{ }^{*}=p<0.01$, $* * *=p<0.001$, alb-myct ${ }^{\text {tg }}$ mice vs. WT controls.

\subsection{MAFLD-Associated Changes in the Liver of Middle-Aged alb-myc ${ }^{\text {tg }}$ Mice}

Recent studies support a central role of MS in the pathogenesis of MAFLD [13]. We did not detect any significant differences in liver weight or in hepatosomatic ratio between transgenic and WT mice (Figure S5A,B). However, careful examination of liver histology revealed that alb-myctg mice with ageing are much more prone to develop abnormal accumulation of lipids in the liver compared to control animals. H\&E staining showed microvesicular steatosis characterized by distended hepatocytes with small lipid vesicles and foamy appearing cytoplasm (Figure 3A). These findings were confirmed by Oil-Red-O staining (Figure 3B,C) and by direct quantification of hepatic TG (Figure 3D). 
Blinded quantitative analysis performed by an experienced pathologist revealed that middle-aged alb-myctg mice exhibited micro- and macrovesicular steatosis grade 1 associated with hepatocyte ballooning reaching in most of the animals a 2.4 NAFLD activity score (NAS) (Figure 3E).

A

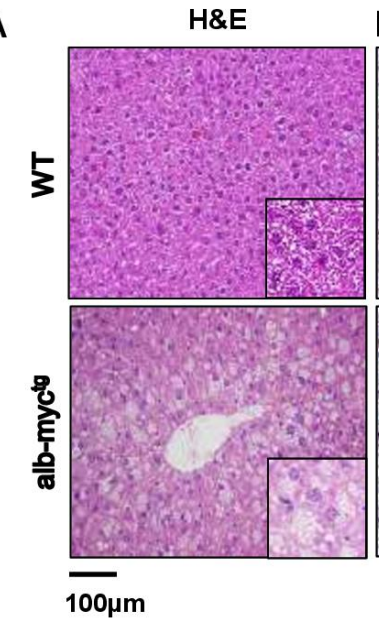

B ORO

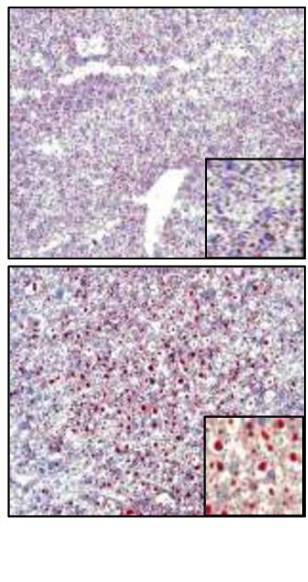

C

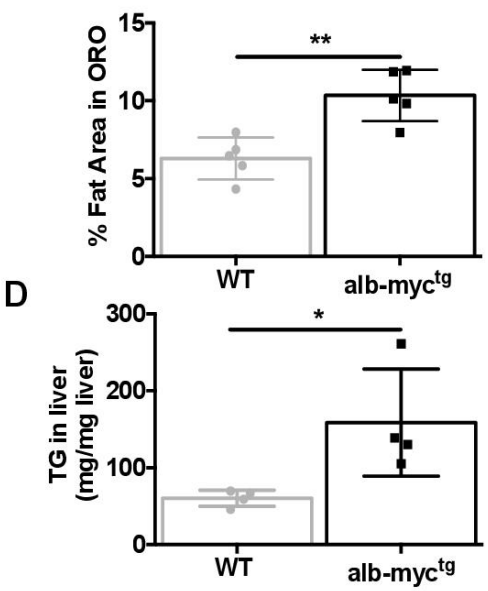

$E$

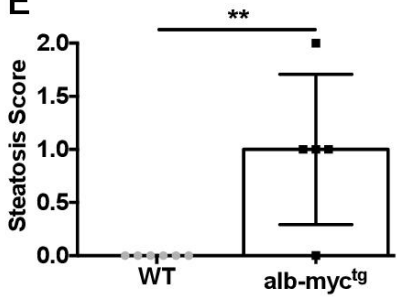

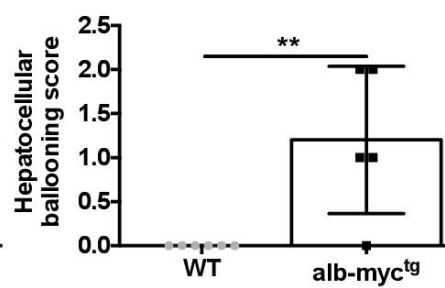

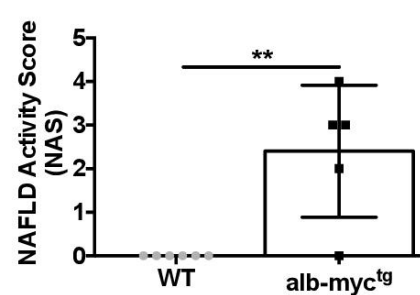

Figure 3. Steatotic changes in the liver of transgenic mice. (A) Representative liver H\&E staining demonstrating distended hepatocytes with foamy appearing cytoplasm and small lipid vesicles. Scale bar $=100 \mu \mathrm{m}(n=8-9)$. (B) Illustrative ORO staining. Scale bar $=100 \mu \mathrm{m}$. (C) Quantitative analysis of ORO-stained area $(n=5)$. (D) Direct TG quantification $(n=4)$. (E) Histology score for steatosis, hepatocyte ballooning, NAFLD activity score (NAS) $(n=5-6)$. Data are expressed as the mean $\pm \mathrm{SD}$, ${ }^{*}=p<0.05 ;{ }^{* *}=p<0.01$, alb-myctg mice vs. WT controls.

Consistent with an abnormal lipid metabolism, the expression of $S c d 1$ gene increased significantly in hepatic tissue from 36-week-old alb-myctg (Figure S6A).

Hence, hepatic accumulation of lipids in transgenic animals leads to compensatory enhancement of mitochondrial $\beta$-oxidation and increased expression of $\mathrm{Cpt} 1$ and Acadm genes (Figure 4A). Obviously, this process is insufficient to normalize lipid levels and in turn generate considerable amounts of reactive oxygen species (ROS) and oxidative stress. Consequently, a lipid peroxidation marker, 4-HNE was increased in the liver parenchyma of alb-myc ${ }^{\text {tg }}$ mice (Figure 4B). Another important cause of ROS overproduction in alb-myctg is CYP2E1 overexpression (Figures $4 \mathrm{C}$ and S6B). Enhanced ROS production triggers the unfolded protein response (UPR) and compensatory Ucp 2 activation (Figure S6C). Excess workload misfolded proteins cause activation of the UPR, presented in c-myctg mice by activation of $\mathrm{CHOP}$ (Figures $4 \mathrm{C}$ and $\mathrm{S6B}$ ).

Concomitant with these data, transmission electron microscopy (TEM) revealed large and aggregated lipid deposition; disorganized and fragmented rough endoplasmic reticulum (ER) in c-MYC transgenic mice (Figure S6D). 
A

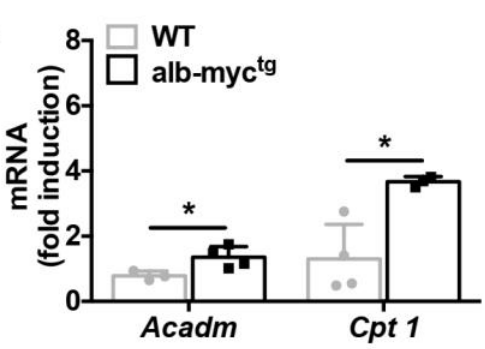

B
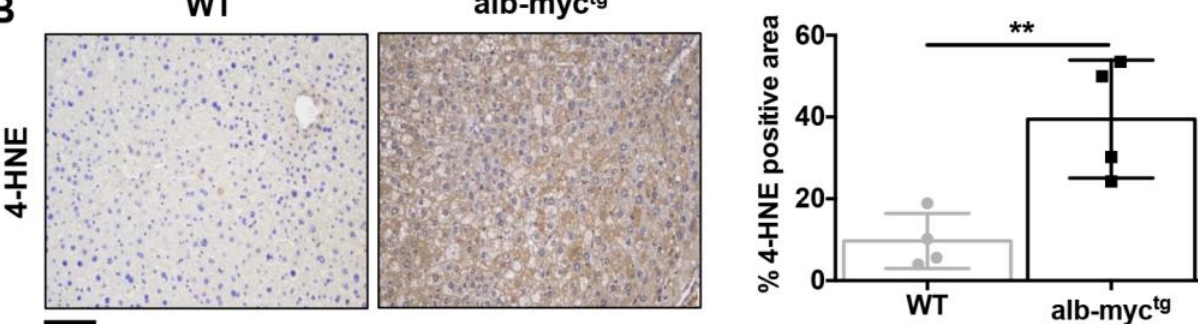

C

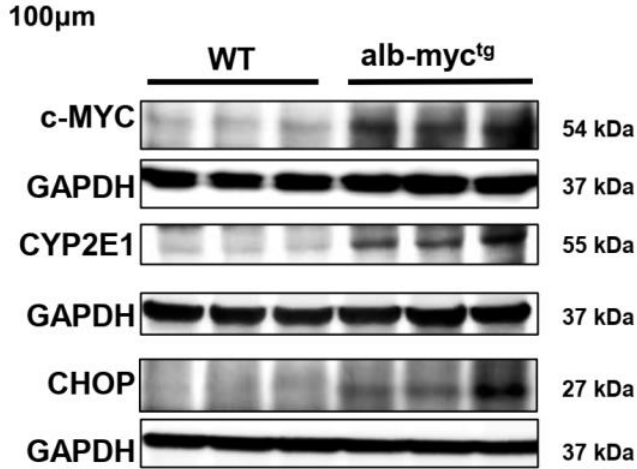

D

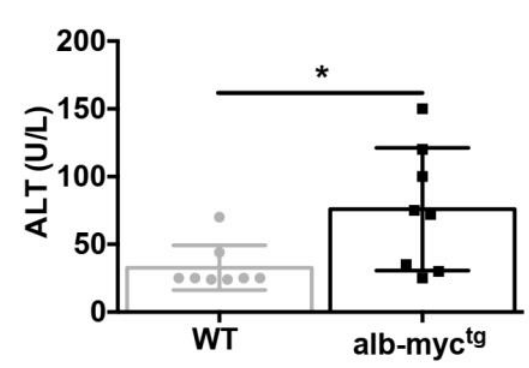

E
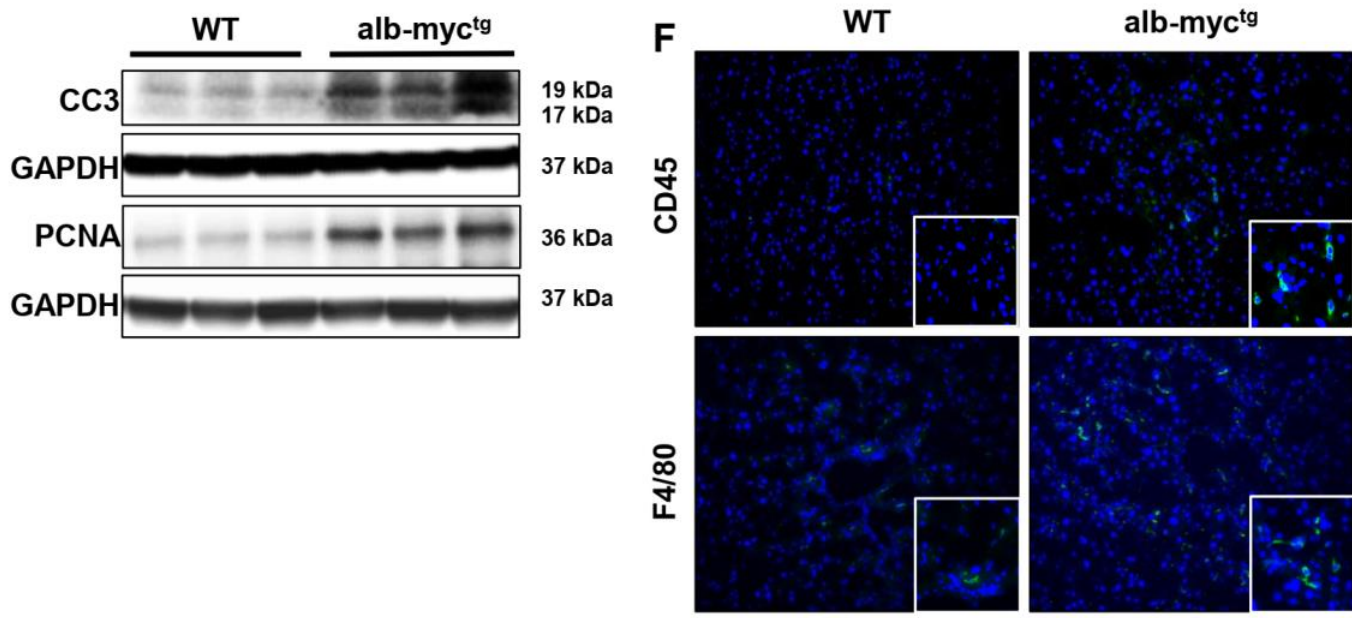

G

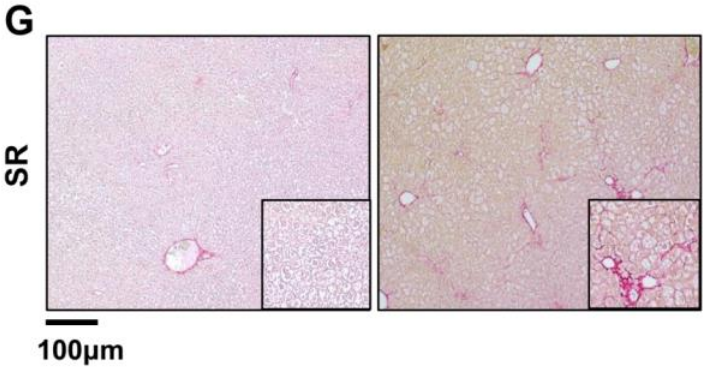

Figure 4. Transgenic overexpression of c-MYC in the livers leads to induction of ER stress, apoptosis, inflammation and fibrosis. (A) qPCR analysis of hepatic mRNA expression of Acadm and Cpt1 genes 
$(n=6)$. (B) 4-HNE expression (brown) of representative livers was evaluated by immunohistochemistry. Scale bar $=100 \mu \mathrm{m}$. The histogram represents the 4-HNE staining intensity (\% square microns) $(n=4)$. (C) Immunoblot analysis of c-MYC, CYP2E1, CHOP $(n=3)$. Uncropped blots are presented in Figure S13. (D) Levels of ALT in serum $(n=8-9)$. (E) Protein expression of CC3 and PCNA $(n=3)$. GAPDH was used as a control for protein loading). Uncropped blots are presented in Figure S14. (F) Representative IF images of hepatic inflammation (CD45 and F4/80 positive cells are stained in green). (G) Sirius red (SR) staining of liver paraffin sections showing accelerated onset of fibrosis formation in alb-myctg. ${ }^{*}=p<0.05 ;{ }^{* *}=p<0.01$.

Prolonged activation of the UPR and ROS production in alb-myct mice likely contributed to liver damage and caused a mild but significant increase of the plasma levels of ALT (Figure 4D), a major clinical indicator of cellular liver injury. Consistently, c-MYC overexpression induced moderate apoptotic cell death in livers of transgenic mice as evidenced by cleaved caspase 3 (CC3) immunoblot (Figures 4E and S7A). Death of hepatocytes triggers compensatory proliferation in surrounding cells to maintain tissue homeostasis (Figure 4E). The analysis of PCNA (Figures 4E and S7A) and Ki-67 (Figure S7B) expression revealed moderate hepatic proliferation in the livers from alb-myc ${ }^{\mathrm{tg}}$ mice.

Hepatic steatosis and cell death in the liver further caused immune cell infiltration and hepatic inflammation [14]. Alb-myctg animals showed an increased accumulation of CD45- and F4/80-myeloid positive cells as assessed by IF staining (Figures 4F and S7C,D). Finally, analysis of liver fibrosis, the hallmark of $\mathrm{SH}$, revealed marked fibrosis in livers from alb-myct ${ }^{\mathrm{tg}}$ mice by SR staining (Figures $4 \mathrm{G}$ and S7E).

Increased glutamine catabolism is a major metabolic feature of rapidly proliferating cells such as hepatocytes and $\alpha \mathrm{SMA}$-positive myofibroblasts. Indeed, c-MYC has been identified as an upstream regulator of GLS1 [15]. Therefore, we found increased expression of glutaminase 1 (GLS1) and glutamine synthetase (GS) in the livers of alb-myct mice (Figure S8A,B).

Finally, gene array data in the livers of alb-myctg mice under basal conditions revealed upregulation of pathways related to cytochrome P450 and ER-stress response, lipid/fatty acid metabolism, glucose homeostasis, DNA damage and proliferation/cell cycle, in accordance with previous publications [10] (Figure S9A-D).

In agreement with previous reports [16], 20\% of transgenic mice at the age of 52 weeks develop liver tumor (Figure S10).

In summary, our data strongly suggest an important role of the oncogene c-MYC in murine livers for MAFLD initiation and subsequent tumor promotion.

\subsection{WD Feeding Accelerates Liver Injury, Steatohepatitis, Fibrosis and Initiation of MAFLD Associated HCC in alb-mycts Mice}

Next, we fed our transgenic model a WD using 12-week-old alb-myc ${ }^{\text {tg }}$ and WT mice for 24 weeks. alb-myc ${ }^{\text {tg }}$ mice fed with WD did not gain weight faster (Figure S11A), however; they developed higher BMI compared to WT mice at the experimental end-point (Figure 5A,B). This was accompanied by hypertrophy and inflammation of adipocytes and eWAT inflammation (Figures $5 B$ and S11B,C). Additionally, transgenic mice showed significant upregulation of blood glucose after fasting, increase in total serum cholesterol and hypertriglyceridemia (Figure 5C). WD induced micro- and macrovesicular steatosis in both groups of mice. However, the level of steatosis was much stronger in transgenic mice compare to WT littermates (Figures 5D and S11D). This was further confirmed by measurement of hepatic TG (Figure 5D, right) and by pathological evaluation of NAFLD activity (Figure S11E). Consistently, WD-fed alb-myctg mice 24 weeks of exhibited stronger hepatic inflammation (CD45 positive cells) (Figures 5E and S11F) and extensive hepatic fibrosis, assessed by SR staining (Figures $5 \mathrm{~F}$ and S11G) and $\alpha \mathrm{SMA}$ (Figures $5 \mathrm{G}$, middle and S11H,I) expression. SH in the liver was accompanied by high compensatory proliferation, confirmed by elevated numbers of Ki-67 positive cells (Figures 5H, right and S11J) and apoptotic cell death, detected by CC3 overexpression (Figures 5I and S11K). 


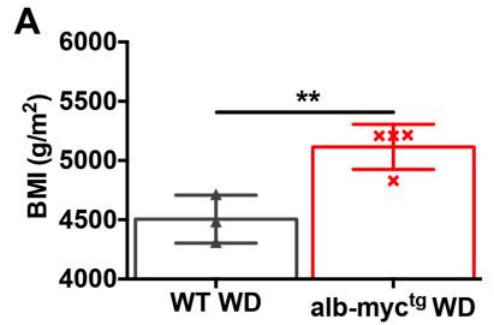

C

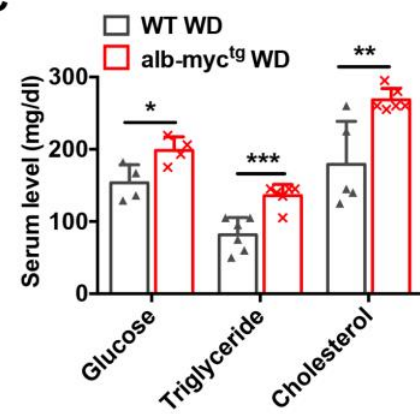

D

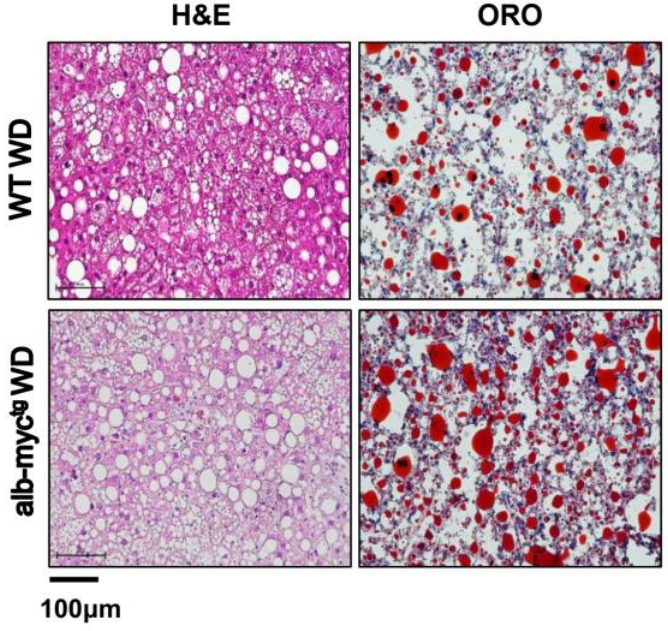

B

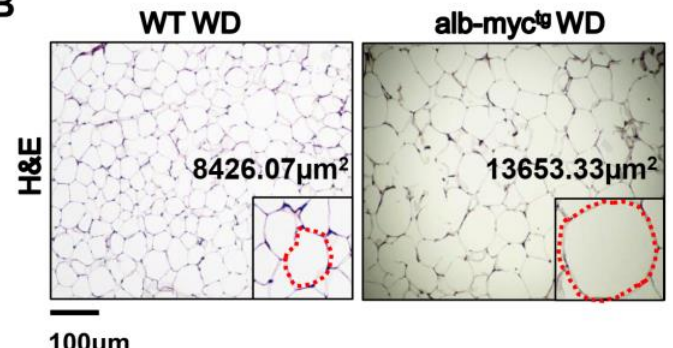

E
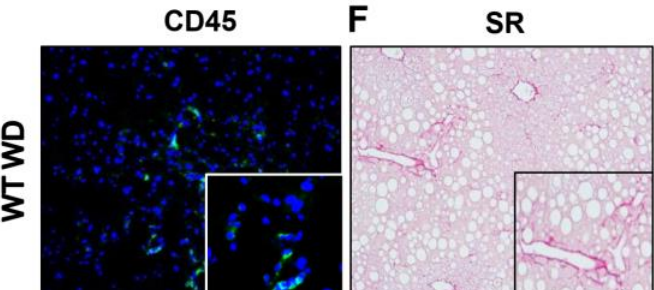

G aSMA

H Ki-67
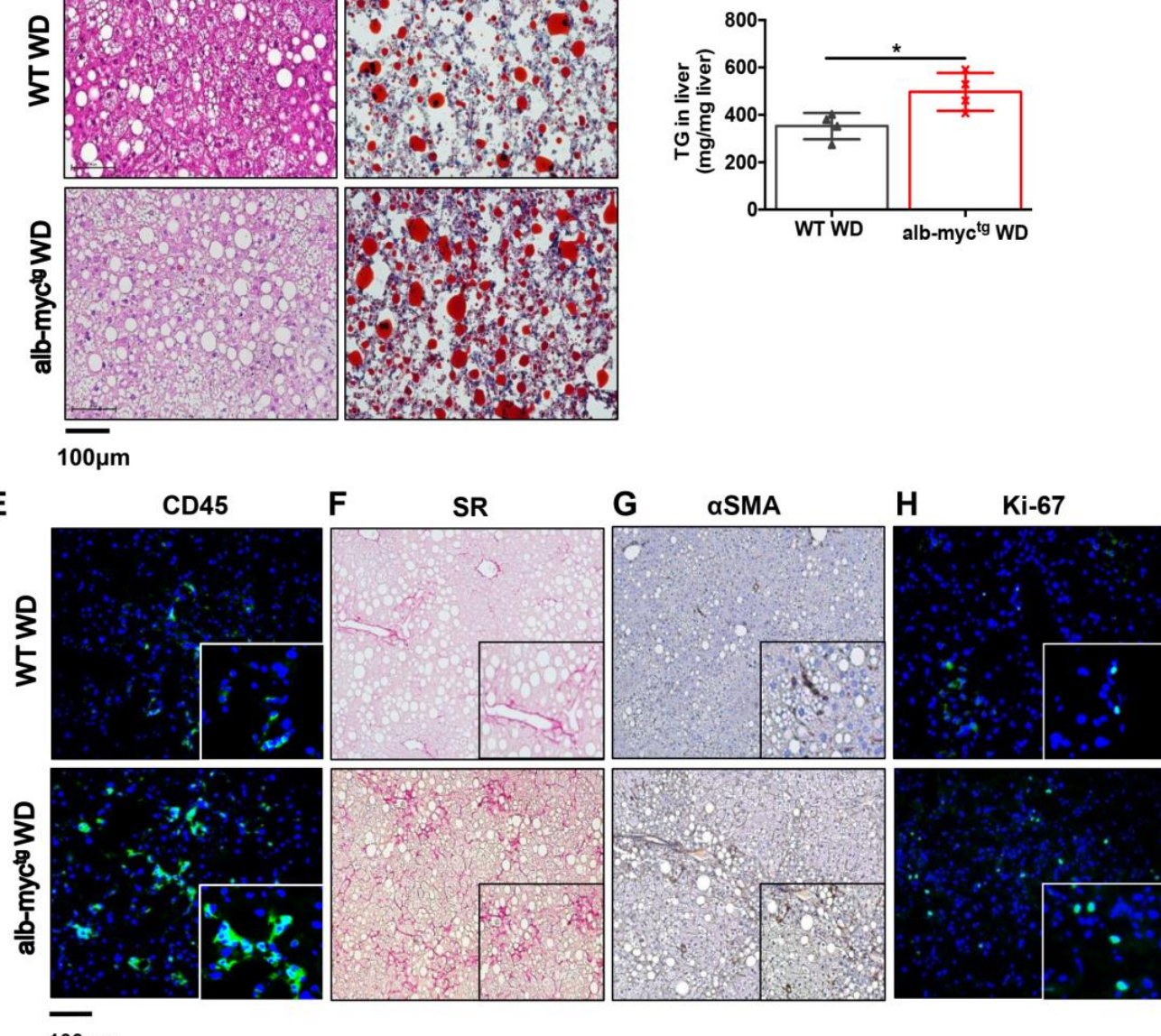

I

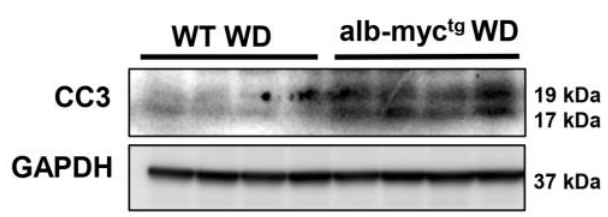

Figure 5. WD feeding accelerates liver injury in alb-myct ${ }^{\mathrm{g}}$ mice. WT $(n=6)$ and alb-myc ${ }^{\text {tg }}(n=7)$ mice 
fed for 24 weeks with WD. (A) Body mass index (BMI) $\left(\mathrm{g} / \mathrm{m}^{2}\right)$ of mice was calculated at the age of 36 weeks $(n=3-4)$. (B) H\&E staining of epididymal white adipose tissue (eWAT). The dotted red line marks the individual adipocyte. Scale bar $=100 \mu \mathrm{m}$. (C) Fasting blood glucose, levels of cholesterol and TG in serum $(n=6-8)$. (D) Representative liver sections stained with H\&E and ORO are shown. Scale bar $=100 \mu \mathrm{m}$. Histogram showing TG quantification of mice fed WD $(n=4)$. (E) Representative images of hepatic inflammation (CD45 positive cells stained in green). (F,G) Liver fibrosis (SR and $\alpha$ SMA IHC staining). (H) Hepatic proliferation (Ki-67 positive cells in green). Scale bar $=100 \mu \mathrm{m}$. (I) Protein expression of cleaved caspase 3, GAPDH used as loading control $(n=3)$. Uncropped blots are presented in Figure S15. Data are expressed as the mean \pm SD, ${ }^{*}=p<0.05$, ${ }^{* *}=p<0.01,{ }^{* * *}=p<0.001$, alb-myc ${ }^{\text {tg }}$ mice fed with WD compared to WT fed with WD.

Finally, with the chronic feeding with a WD for a period of 40 weeks led to the development of multiple tumor foci in $100 \%$ alb-myctg mice (five out of five). In contrast, none of the WT animals fed with WD had visible tumor nodules at this time point (zero out of five) (Figure 6A).

H\&E (Figure 6B) and SR (Figure 6C) of the liver demonstrated well demarcated nodules surrounded by extensive collagen deposition. Moreover, massive accumulation of highly proliferative Ki-67 positive cells was detected inside the nodules (Figure 6D). Importantly, these changes were accompanied by extensive c-MYC nuclear expression in the liver tissue of transgenic mice (Figure 6E).

Altogether, our data strongly support the hypothesis that oncogene c-MYC and WD synergistically drive MAFLD progression towards carcinogenesis.

\subsection{Anti-MAFLD and MS Potential of Metformin in alb-myctg Mice}

Metformin, an oral biguanide is now generally accepted as the first-line treatment for DM2. Moreover, metformin has demonstrated antineoplastic effects in several types of tumors, including HCC $[17,18]$. Since DM2 and IR play the key roles in the pathogenesis of MAFLD, we next evaluated the effects of metformin treatment in c-MYC transgenic mice.

Alb-myctg fed with chow diet enriched with $0.1 \%$ metformin gained significantly less weight (Figure S12A,B) and exhibited significantly lower BMI (Figure 7A) at experimental end-point (36 weeks) in comparison with alb-myc ${ }^{\text {tg }}$ mice fed with chow diet. Importantly, metformin-enriched chow diet led to considerable decrease of fasting glycaemia as well as improved glucose tolerance in GTT (Figure 7B).

Transgenic mice fed with met+chow diet accumulated less adipose eWAT tissue (Figure 7C) and demonstrated improved parameters of lipidemia (TG and cholesterol in blood) (Figure 7D). Remarkably, metformin-enriched chow diet improved hepatic steatosis (Figure 7E,F), attenuated collagen accumulation (Figure 7G), decreased AST level (Figure S12C) and mildly improved hepatic proliferation (Figures 7H and S12D) in transgenic animals. Importantly, these positive changes were not a consequence of a direct inhibition of c-MYC expression (Figure S12E), but rather effects of diminished lipogenic sterol-regulated element-binding protein (SREBP1) overexpression (Figures 7I and S12F) in alb-myc ${ }^{\text {tg }}$ after metformin treatment.

Altogether, our data showed the efficacy of metformin on lipogenic targeting in c-MYC transgenic mice leading to positive histological and biochemical alterations. 
A

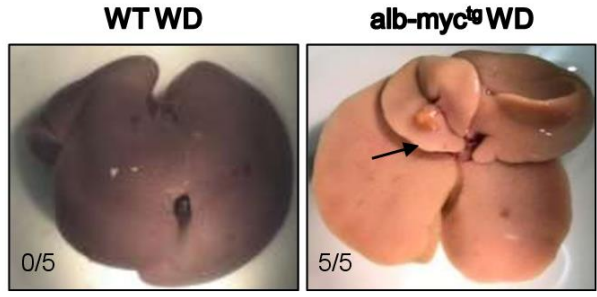

B

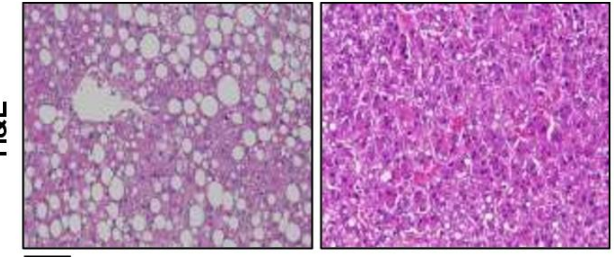

C

$$
\overline{100 \mu \mathrm{m}}
$$
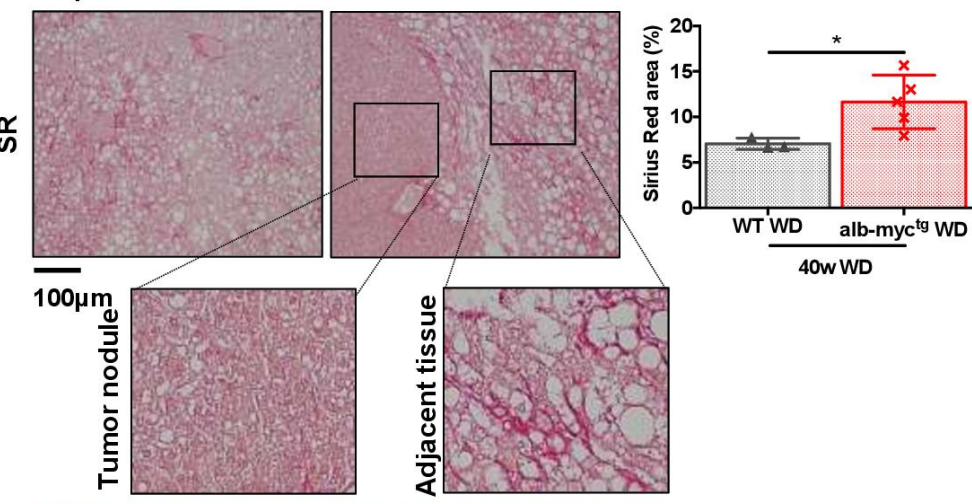

D
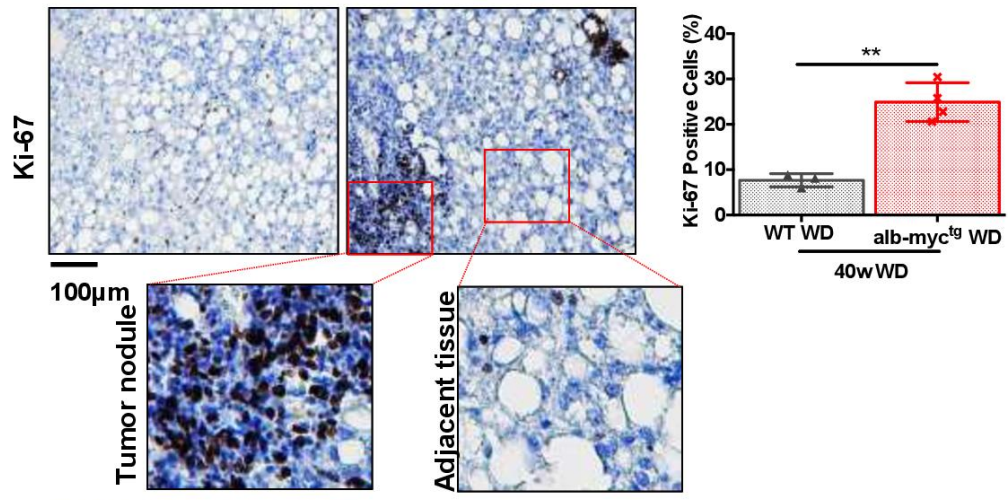

$E$
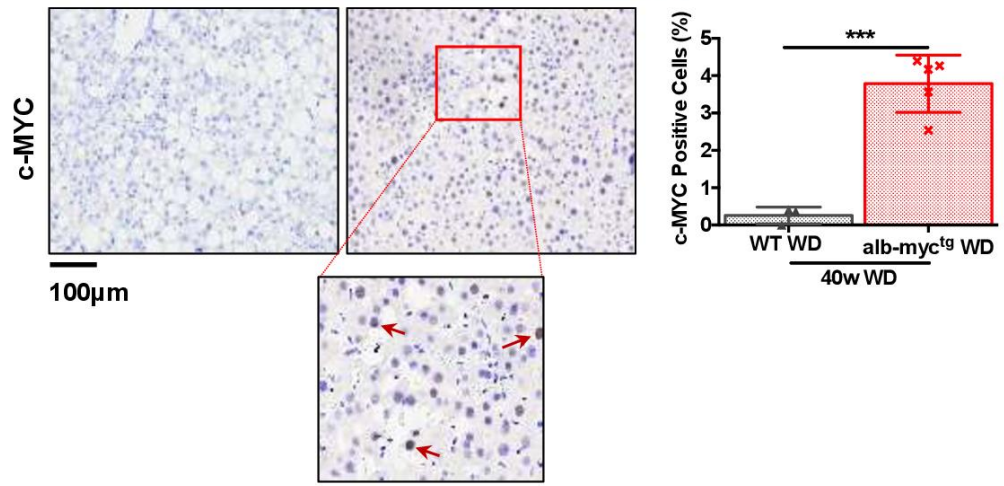

Figure 6. WD feeding accelerates the initiation of MAFLD associated tumorigenesis in alb-myc ${ }^{\text {tg }}$ mice. WT $(n=3)$ and alb-myctg $(n=5)$ mice fed 40 weeks with WD. (A) Gross liver. Numbers represent tumor incidence. (B) Representative liver sections stained with H\&E. (C) Representative liver sections stained with SR. Quantification of $\%$ SR positive area $(n=3-5)$ Scale bar $=100 \mu \mathrm{m}$. (D) Ki-67 immunostaining of paraffin sections showing increased cell proliferation (brown) of hepatocytes in 
alb-myctg mice liver. Quantification of Ki-67 positive cells $(n=3-5)$. Scale bar $=100 \mu \mathrm{m}$. (E) c-MYC IHC staining of paraffin sections. Quantification of c-MYC positive cells $(n=3-5)$. Scale bar $=100 \mu \mathrm{m}$. ${ }^{*}=p<0.05,{ }^{* *}=p<0.01,{ }^{* * *}=p<0.001$.

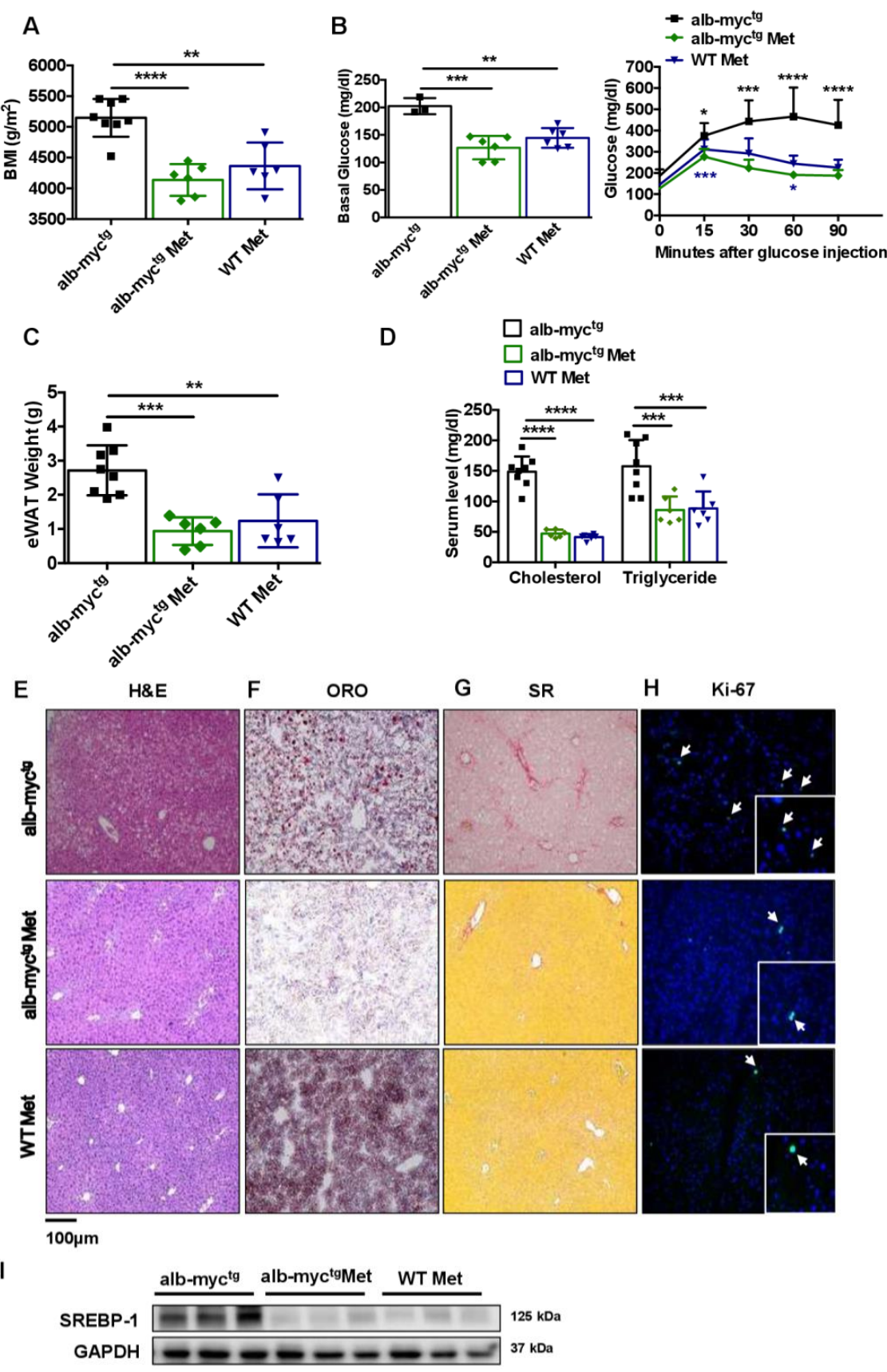

Figure 7. Improved hepatic phenotype in alb-myctg mice after metformin treatment. alb-myctg mice $(n=6)$ and WT mice $(n=6)$ fed 20 weeks received metformin enriched chow diet, alb-myctg $(n=8)$ received chow diet; all animals were sacrificed at the age of 36 weeks. (A) Body mass index (BMI) $\left(\mathrm{g} / \mathrm{m}^{2}\right)$ of mice at the age of 36 weeks $(n=3-6)$. (B) Basal glucose level after $6 \mathrm{~h}$ fasting. Blood glucose concentration curve during intraperitoneal GTT. $(n=3-6)$. (C) eWAT weight $(n=6-8)$. (D) Serum levels of cholesterol and TG in serum $(n=6-8)$. Representative liver sections stained with H\&E (E), ORO (F), SR (G) and Ki-67 (H) stainings. Scale bar $=100 \mu \mathrm{m}$. (I) Protein expression of SREBP-1, GAPDH used as loading control $(n=3)$. Uncropped blots are presented in Figure S16. Data are expressed as the mean $\pm \mathrm{SD},{ }^{*}=p<0.05,{ }^{* *}=p<0.01,{ }^{* * *}=p<0.001,{ }^{* * * *}=p<0.0001$, alb-myc ${ }^{\mathrm{tg}}$ mice fed metformin enriched chow diet vs. alb-myctg mice fed chow diet; ${ }^{* *}=p<0.01,{ }^{* * *}=p<0.001$, ${ }^{* * * *}=p<0.0001$, WT mice fed metformin enriched chow diet vs. alb-myctg mice fed chow diet. 


\section{Discussion}

c-MYC, "the oncogene from hell", is associated with more than half of all human cancers [19]. Gains of chromosome 8q22-24 region, encoding c-MYC are amongst the earliest genomic events associated with liver cancer development [20]. According to the Cancer Genome Atlas Research Network c-MYC alterations are reported in up to $12 \%$ of HCC [12]. Novel data suggest that deregulation of c-MYC function is not only associated with HCC development, but also with CLD (e.g., ALD, viral hepatitis, liver fibrosis/cirrhosis, for hepatoblastoma and cholangiocarcinoma, etc.) [8].

Since defective-MYC expression is an early event in carcinogenesis, the characterization of the consequences of c-MYC overexpression in hepatocytes is of ultimate interest. Indeed, hepatocyte-specific alb-myc ${ }^{\text {tg }}$ mice are predisposed to HCC albeit a long period of latency $[11,21] .20 \%$ alb-myct mice at the age of 52 weeks displayed liver tumors that were mostly mono- or two-nodular. The gene signature of these tumors exactly resembles slow growing human HCC [16,22].

Hence, the oncogene c-MYC, "super-transcription factor", is not only a master regulator of cell growth and cell cycle arrest, but also plays an essential role in the regulation of metabolic reprogramming. Proliferating cells change their metabolic demands compared to quiescent cells as only global metabolic alterations permit tumor cells to survive and proliferate despite adverse conditions [23,24].

Our experiments revealed that middle-aged c-MYC transgenic mice on standard chow diet are prone to develop abnormal accumulation of lipids in the liver compared to control mice. Consistently, gene expression profile of alb-myc ${ }^{\text {tg }}$ revealed significant alterations in FA metabolism. Activation of lipid synthesis is very important for fast growing cells, because lipids are fundamental for functions such as membrane generation, protein modification and bioenergetics requirements. In a wide variety of tumors, de novo FA synthesis is activated independently of the levels of circulating lipids [25]. The main product of FA synthesis in the cytoplasm is saturated palmitic acid, formed by rate-limiting enzyme-SCD1—on the cytosolic side of the ER. Consequently, SCD was upregulated in alb-myctg livers. Hence, SCD1 overexpression has been reported as promotor in liver fibrosis and tumor development [26].

FA overload in c-MYC transgenic hepatocytes acted as both a substrate and an inducer of microsomal cytochrome P-450 (CYP2E1) and fatty acid oxidation systems (Cpt1, Acadm) that generate reactive oxygen species resulting in oxidative stress (20). Lipid peroxidation products as well as proteins modified by ROS develop inflammatory response and lead to immune cells $\left(\mathrm{CD} 45^{+}, \mathrm{F} 4 / 80^{+}\right)$infiltration into the hepatic parenchyma. Inflammatory cytokines, produced by immune cells further activate HSCs and stimulate the production of collagen fibers and ECM deposition in the liver, leading to fibrogenesis [27].

TG excess in the serum of alb-myctg mice represent the mechanism by which FA are exported from the livers and delivered to adipose tissue for storage [28]. Hence, 36 weeks old transgenic animals developed considerably greater weight gain, BMI and e-WAT increase compare to control littermates on normal chow diet. Visceral adiposity and low-grade state of adipose tissue inflammation, assessed by presence of macrophage CLS in WAT, contribute to IR and hyperglycemia in transgenic mice [29,30]. High blood glucose level results in IR, and further contributes to metabolic disorders in the liver.

Tumor cells take in more glucose than normal to elevate aerobic glycolysis with the Warburg effect. Gene array data showed upregulation of PFKFB1-3 in transgenic liver, a key enzyme regulating glucose metabolism and promoting aerobic glycolysis. Hence, previous publications have shown that inhibitors targeting PFKFB3 have been found to suppress aerobic glycolysis, decrease glucose uptake, and induce cancer cell autophagy [31]. Moreover, consistently with previous publications transgenic expression of c-MYC also enhances the expression of mitochondrial GL and GLS for canonical glutaminolysis. Glutamine is the most abundant and nonessential amino acid that can be synthesized from glucose. Proliferative cells in many cancers, including some hepatocellular carcinomas, are glutamine addicted, and must induce GLS1 to increase glutamine catabolism and fuel 
their growth $[32,33]$. Interestingly, the hepatic GLS1 is also overexpressed in MAFLD patients [34].

Altogether, overexpression of c-MYC exclusively in the liver affects the whole-body metabolism and leads to the development of mild steatohepatitis/fibrosis in transgenic mice.

However, the development of MAFLD as well as further progression to HCC depends on multiple parallel factors, acting synergistically in genetically predisposed individuals. Thus, in order to evaluate a putative role of c-MYC in cancer initiation and progression we next intensified our model and applied high caloric WD to alb-myc ${ }^{\text {tg }}$ mice. In fact, this model will mimic the situation in human MAFLD, there combination of endogenous (e.g., oncogenes) and exogenous (food habits) factors synergistically contribute to the development of HCC.

As expected, c-MYC overexpression exacerbated steatosis, liver injury, liver inflammation, fibrosis and strong compensatory proliferation in transgenic mice fed with WD. Importantly, after 40 weeks of feeding all transgenic animals developed foci of tumors.

These findings are consistent with previously reported earlier HCC enhancement in c-MYC transgenic animals fed with either methionine choline-deficient diet (MCD) or choline-deficient and amino acid-defined diet (CDAA) [35]. Hence, we can conclude that metabolic changes induced by c-MYC promote hepatocarcinogenesis in a series of different murine MAFLD and HCC models.

Does the c-MYC transgenic mouse model reflect the clinical reality of human MAFLDassociated HCC? To answer this question, we analyzed human liver biopsy samples with MAFLD and MAFLD-associated HCC. Although a small cohort of 11 samples was used, we found an increased expression and prominent nuclear localization of the proto-oncogene c-MYC in patients with MAFLD and MAFLD-related HCC, highlighting its potentially critical role for the disease progression. Future studies with expanded sample size will needed to further confirm the role of c-MYC in human MAFLD development.

The limitations of a curative pharmacological treatment for human HCC clearly underline the need for the identification of novel target molecules. The MAFLD- HCC sequence suggest that specific inhibitors against metabolic signaling pathways may allow to interrupt the continuous transition from CLD to cancer.

Hence, in the current study we supplemented the chow diet with metformin and applied it to c-MYC transgenic animals. The use of metformin, the most commonly prescribed drug for DM2, was repeatedly associated with the decreased risk of the occurrence of various types of cancers, especially of pancreas and colon and HCC. This observation was also confirmed by the results of numerous meta-analyses that confirmed that metformin reduces cancer incidence by 30-50\% [36]. Several previous publications reported MYC inhibition by metformin $[37,38]$. In the current study we could not detect the direct inhibition of c-MYC in the liver of transgenic mice by metformin-enriched diet. Hence we found that metformin had a strong inhibitory effects on SREBP1 expression in alb-myctg animals. Due to the lipogenic inhibition in the liver, alb-myctg mice treated with metformin diet were resistant to obesity, had modestly improved glucose and lipid metabolic parameters, and exhibited a marked lowering of liver steatosis and overall reduced collagen accumulation.

Our findings are consistent with previous reports showing the interaction of c-MYC with SREBP1 $[39,40]$. Indeed, as highly proliferating cancer cells require enhanced lipid production, c-MYC induces SREBP and together they collaborate to activate fatty acid (FA) synthesis and drive FA elongation from glucose and glutamine [41,42]. These findings point toward the promise of blocking c-MYC induced de novo lipogenesis as a general therapeutic strategy to combat common MYC-driven cancers. Hence in spite of remarkable improvement of steatohepatitis in alb-myc ${ }^{\text {tg }}$ mice treated with metformin, we could detect only mild changes in hepatic proliferation. Therefore, future studies will need to address the intriguing possibility of metformin to influence MYC-mediated cell growth.

In summary, phenotypical changes in the livers derived by c-MYC oncogene closely recapitulate the human MAFLD, albeit in a more telescope time frame. Hence, the evaluation of the molecular mechanisms underlying the associations between new genetic 
risk factors and progressive liver disease could be useful for the identification of novel therapeutic targets for MAFLD. Importantly, the detection of such preexisting risk factors should be used to identify the "high risk" MAFLD patients in whom preventive measures such as regular individual counselling and screening must be undertaken [41]. In fact, life expectancy of patients with HCC depends on the stage of the cancer at diagnosis. In advanced stages, some months are expected; however, if diagnosed in early stages, curative treatments such as surgical resection, liver transplant and local ablation can improve the survival of the patients and a 5-year survival rate can be reached. A very recent study demonstrated that the shorter survival of MAFLD-HCC patients, compared to HCV-HCC patients, is mainly due to the late diagnosis and greater tumor burden and not because $\mathrm{HCC}$ in MAFLD is more aggressive [42].

\section{Conclusions}

The current study, based on a clear and novel phenotype of transgenic mice, as well as interesting findings in patients, makes c-MYC an attractive target which can help to guide physicians towards preventative strategies and individualized therapy that can improve clinical outcome of MAFLD-related HCC.

Supplementary Materials: The following supporting information can be downloaded at: https:/ / www.mdpi.com/article/10.3390/cancers14010192/s1, Figure S1: Bioinformatic analysis of 353 human HCC samples based on data generated by the Cancer Genome Atlas (TCGA) Research Network, Figure S2: Representative IHC-stained sections demonstrating nuclear expression of c-MYC. (A) c-MYC nuclear expression inside tumor nodules in patients with MAFLD-related HCC. Left: the DAB-stained images. Right: Hematoxylin stained images. (B) c-MYC nuclear expression in MAFLD patients with advanced liver fibrosis. Left: the DAB-stained images. Right: Hematoxylin stained images, Figure S3: MS in alb-myctg at baseline. WT $(n=9)$ and alb-myctg $(n=8)$ mice at the age of 36 weeks. (A) Food caloric intake was stable in both groups through the duration of study. (B) Representative MRI scan images of WT and alb-myc ${ }^{\text {tg }}$ mice showing body fat distribution in 26 weeks old animals. The quantitative data represent fat/body ratio (\%) means. (C) Representative F4/80 IF staining of WAT. Positive cells are stained in green and marked with red arrows. Nuclei are stained in blue using DAPI as counterstain. Scale bar is $100 \mu \mathrm{m}(n=4)$, Figure S4: Glucose intolerance. (A) The histogram represents the incremental area under the respective glucose tolerance curve $(n=3)$. (B) Insulin tolerance test (ITT), and the histogram represents the incremental area under the curve $(n=3)$. Data are expressed as the mean $\pm \mathrm{SD},^{*}=p<0.05 ;{ }^{* *}=p<0.01$, alb-myc ${ }^{\text {tg }}$ mice vs. WT controls, Figure S5: Hepatosomatic ratio at 36 weeks. WT $(n=9)$ and alb-myctg $(n=8)$ mice at the age of 36 weeks. (A) Liver weight. (B) Liver to body weight ratio, Figure S6: Hepatic phenotype in WT and alb-myctg mice at 36 weeks. (A) qPCR analysis of hepatic mRNA expression expression of Scd1. (B) Histograms showing densitometric analysis of western blots for c-MYC, CYP2E1 and CHOP. (C) qPCR analysis of hepatic mRNA expression expression of $U c p 2(n=4)$. (D) Representative TEM images of hepatic endoplasmic reticulum. Representative Transmission Electro-Microscopy (TEM) pictures of WT and alb-myc ${ }^{\text {tg }}$ mice. Asterisks indicate fat droplets in hepatocytes. Arrows mark endoplasmic reticulum (ER). Scale $=10 \mu \mathrm{m}$ and $1 \mu \mathrm{m}$, Figure S7: Spontaneous proliferation and mild inflammation in alb-myctg mice at 36 weeks. (A) Histograms showing densitometric analysis of western blots for CC3 and PCNA. (B) Ki-67 immunofluorescence of crytosections showing increased cell proliferation (green, as indicated by arrow) of hepatocytes in alb-myc ${ }^{\text {tg }}$ mice liver and the quantification of $\% \mathrm{Ki}-67$ positive cells $(n=8)$. (C-E) Quantification of \% CD45, F4/80- positive cells and SR area respectively using ImageJ software $(n=8)$. Data are expressed as the mean $\pm \mathrm{SD},{ }^{*}=p<0.05 ;{ }^{* *}=p<0.01,{ }^{* * *}=p<0.001$, alb-myctg mice vs. WT controls, Figure S8: Increased glutamine catabolism in alb-myctg mice at 36 weeks. $(n=5)$. (A) IHC staining of Glutamine synthetase (GS). (B) Protein expression of Glutaminase 1 (GLS1) in the livers of alb-myctg and WT mice, Figure S9: Activation of signaling pathways relevant to human MAFLD in the liver of mice. (A) Dysregulated genes involved in different pathways. (B) Heat map demonstrating deregulated genes. Red and green colors indicate high and low gene expression, respectively. (C) Top canonical pathways. (D) Molecules involved in hepatotoxicity. All data are based on statistical significance, Figure S10: Spontaneous HCC development in alb-myc ${ }^{\mathrm{tg}}$ mice. WT $(n=3)$ and alb-myctg $(n=5)$ mice at the age of 52 weeks. (A) Macroscopic images of the liver. Numbers represent tumor incidence (\%) means (B) Representative liver sections 
stained with H\&E, Figure S11: Phenotypical changes in WT and transgenic mice fed with WD for 24 weeks. (A) Growth curve demonstrating body weight gain, assessed at 7 days interval at each group (B) Relevant adipocyte area of eWAT $(n=6)$. (C) Representative F4/80 IF staining of WAT. Positive cells are stained in green and marked with red arrows. Nuclei are stained in blue using DAPI as counterstain. Scale bar is $100 \mu \mathrm{m}(n=4)$. (D) Quantification of Oil Red O positive area $(n=5)$. (E) Histological NAFLD activity score $(n=5)$. (F) Quantification of \% CD45 positive cells $(n=6)$. (G) Quantification of \% SR positive area $(n=4)$. (H) Quantification of $\% \alpha$ SMA positive area $(n=3)$. (I) qPCR analysis of hepatic tissue of fibrosis gene $\alpha \operatorname{Sma}(n=6)$. (J) Quantification of \% KI-67 positive cells $(n=6)$. (K) Histograms showing densitometric analysis of western blot for CC3. Data are expressed as the mean $\pm \mathrm{SD},{ }^{*}=p<0.05{ }^{* *}=p<0.01,{ }^{* * *}=p<0.001$, alb-myc ${ }^{\text {tg }}$ mice vs. WT animals fed WD, Figure S12: MS and phenotypical changes in animals fed with metformin enriched diet. (A) Growth curve demonstrating body weight gain of mice, assessed at 7 days interval at each group, and (B) Relevant histogram represents the incremental area under the respective body weight curve. (C) Level of AST in serum $(n=6-8)$. (D) Quantification of \% Ki-67 positive cells $(n=6)$. (E) qPCR analysis of hepatic mRNA expression expression of $c-M y c$. (F) Histograms showing densitometric analysis of western blots for SREBP1. Data are expressed as the mean $\pm \mathrm{SD},{ }^{*}=p<0.05,{ }^{* *}=p<0.01$, ${ }^{* * *}=p<0.001$, alb-myc ${ }^{\text {tg }}$ mice fed metformin enriched chow diet vs. alb-myc ${ }^{\text {tg }}$ mice fed chow diet; ${ }^{*}=p<0.05,{ }^{* *}=p<0.01,{ }^{* * *}=p<0.001,{ }^{* * * *}=p<0.0001$, WT mice fed metformin enriched chow diet vs. alb-myc ${ }^{\text {tg }}$ mice fed chow diet, Figure S13: Uncropped blots for Figure 4C, Figure S14: Uncropped blots for Figure 4E, Figure S15: Uncropped blots for Figure 5I, Figure S16: Uncropped blots for Figure 7I, Table S1: Etiology of human liver samples used for c-MYC gene expression analysis, Table S2: Clinical features of human liver samples, Table S3: Kcal\% of chow diet (CD) and western diet (WD), Table S4: Diets used for the development of MAFLD, Table S5: Antibodies used for IF staining, Table S6: Antibodies used for IHC and IF stainings, Table S7: Primers for qPCR, Table S8: List of antibodies used for WB.

Author Contributions: F.G. performed and analyzed the experiments, acquired the data and drafted the manuscript. O.E.-V. performed and analyzed crucial experiments and acquired the data, R.B.-U., K.Z., C.C., M.M.W., A.L.-P., J.S. (Jorge Simon), T.C.D., M.L.M.-C. contributed by performing crucial experiments. M.G.d.M. helped with TEM. J.S. performed the analysis of gene array. K.P., I.U.E. performed the histopathological analysis. J.R. and T.B. help with the biochemical evaluations. J.V., J.R.R., R.B., C.T., C.L. provided fundamental insights and helped with the manuscript draft. J.S. (Jie Sun) analyzed gene array data. D.M.-M., R.G.-D., Á.R., J.A. and M.R.-G. performed the analysis of human samples. F.J.C. supervised the study and provided funds. Y.A.N. conceived and supervised this project, drafted the paper and provided funds. All authors critically reviewed the manuscript. All authors have read and agreed to the published version of the manuscript.

Funding: This work was supported by the MINECO Retos SAF2016-78711, SAF2017-87919-R, PID2020-117827RB-IOO, PID2020-117941RB-IOO, PID2020-117116RB-I00, EXOHEP-CM S2017/BMD3727, NanoLiver-CM Y2018/NMT-4949, AMMF 2018/117, COST Action CA17112 and UCM-25/2019, La Caixa Foundation Program HR17-00601; Asociación Española Contra el Cáncer (AECC PROYE20084 REGU); the German Research Foundation (SFB1382 Project ID 403224013/A02). FJC and YAN are Ramón y Cajal Researchers RYC-2014-15242 and RYC-2015-17438, respectively. FJC is a Gilead Liver Research Scholar. The research group belongs to the validated Research Groups Ref. 970935 "Liver Pathophysiology", 920631 "Lymphocyte immunobiology", 920361 "Inmunogenética e inmunología de las mucosas" and IBL-6 (imas12-associated). FG and KZ are Chinese Scholarship Council (CSC) fellows. O.E.-V is supported by Beca FPI associated to MINECO SAR2017-87919R and R.B.-U by programa de Financiación de Universidad Complutense de Madrid-Banco Santander, CT63/19. DMM contract is supported by CIBEREHD. DMM, MRG, AR, JA and RG receive support from the Andalusian government (Proyectos Estratégicos-Fondos Feder PE-0451-2018) and from the Instituto de Salud Carlos III (PI16/01842, PI19/01404; PI19/00589). TB is supported by the German Research Foundation (SFB1382 Project ID 403224013/B07).

Institutional Review Board Statement: The study was conducted according to the guidelines of the Declaration of Helsinki, and approved by Ethics Committee of the Junta de Andalucía C.P. S2100062-C.I. 0458-N-21 on 12 February 2021).

Informed Consent Statement: Informed consent was obtained from all subjects involved in the study.

Data Availability Statement: Not applicable. 


\begin{abstract}
Acknowledgments: The authors thank the donors and the Hospital Universitario Virgen del RocíoInstituto de Biomedicina de Sevilla Biobank (Andalusian Public Health System Biobank and ISCIIIRed de Biobancos y Biomodelos-ISCIII-PT20/00069) for the human specimens used in this study. We are grateful to $\mathrm{S}$. Thorgeirsson for providing alb-myc ${ }^{\mathrm{tg}}$ mice.
\end{abstract}

Conflicts of Interest: The authors declare no conflict of interest.

\title{
References
}

1. Anstee, Q.M.; Reeves, H.L.; Kotsiliti, E.; Govaere, O.; Heikenwalder, M. From nash to hcc: Current concepts and future challenges. Nat. Rev. Gastroenterol. Hepatol. 2019, 16, 411-428. [CrossRef] [PubMed]

2. $\quad$ Pais, R.; Barritt, A.S.t.; Calmus, Y.; Scatton, O.; Runge, T.; Lebray, P.; Poynard, T.; Ratziu, V.; Conti, F. Nafld and liver transplantation: Current burden and expected challenges. J. Hepatol. 2016, 65, 1245-1257. [CrossRef]

3. Loomba, R.; Sanyal, A.J. The global nafld epidemic. Nat. Rev. Gastroenterol. Hepatol. 2013, 10, 686-690. [CrossRef] [PubMed]

4. Estes, C.; Razavi, H.; Loomba, R.; Younossi, Z.; Sanyal, A.J. Modeling the epidemic of nonalcoholic fatty liver disease demonstrates an exponential increase in burden of disease. Hepatology 2018, 67, 123-133. [CrossRef]

5. $\quad$ Estes, C.; Anstee, Q.M.; Arias-Loste, M.T.; Bantel, H.; Bellentani, S.; Caballeria, J.; Colombo, M.; Craxi, A.; Crespo, J.; Day, C.P.; et al. Modeling nafld disease burden in china, france, germany, italy, japan, spain, united kingdom, and united states for the period 2016-2030. J. Hepatol. 2018, 69, 896-904. [CrossRef]

6. White, D.L.; Kanwal, F.; El-Serag, H.B. Association between nonalcoholic fatty liver disease and risk for hepatocellular cancer based on systematic review. Clin. Gastroenterol. Hepatol. 2012, 10, 1342-1359 e1342. [CrossRef]

7. Nesbit, C.E.; Tersak, J.M.; Prochownik, E.V. Myc oncogenes and human neoplastic disease. Oncogene 1999, 18, 3004-3016. [CrossRef]

8. Zheng, K.; Cubero, F.J.; Nevzorova, Y.A. C-myc-making liver sick: Role of c-myc in hepatic cell function, homeostasis and disease. Genes 2017, 8, 123. [CrossRef]

9. Nevzorova, Y.A.; Hu, W.; Cubero, F.J.; Haas, U.; Freimuth, J.; Tacke, F.; Trautwein, C.; Liedtke, C. Overexpression of c-myc in hepatocytes promotes activation of hepatic stellate cells and facilitates the onset of liver fibrosis. Biochim. Biophys. Acta 2013, 1832, 1765-1775. [CrossRef]

10. Nevzorova, Y.A.; Cubero, F.J.; Hu, W.; Hao, F.; Haas, U.; Ramadori, P.; Gassler, N.; Hoss, M.; Strnad, P.; Zimmermann, H.W.; et al. Enhanced expression of c-myc in hepatocytes promotes initiation and progression of alcoholic liver disease. J. Hepatol. 2016, 64, 628-640. [CrossRef]

11. Murakami, H.; Sanderson, N.D.; Nagy, P.; Marino, P.A.; Merlino, G.; Thorgeirsson, S.S. Transgenic mouse model for synergistic effects of nuclear oncogenes and growth factors in tumorigenesis: Interaction of c-myc and transforming growth factor alpha in hepatic oncogenesis. Cancer Res. 1993, 53, 1719-1723.

12. The Cancer Genome Atlas Research Network. Comprehensive and integrative genomic characterization of hepatocellular carcinoma. Cell 2017, 169, 1327-1341 e1323. [CrossRef]

13. Marchesini, G.; Marzocchi, R.; Agostini, F.; Bugianesi, E. Nonalcoholic fatty liver disease and the metabolic syndrome. Curr. Opin. Lipidol. 2005, 16, 421-427. [CrossRef]

14. Wang, K. Molecular mechanisms of hepatic apoptosis. Cell Death Dis. 2014, 5, e996. [CrossRef]

15. Tambay, V.; Raymond, V.A.; Bilodeau, M. Myc rules: Leading glutamine metabolism toward a distinct cancer cell phenotype. Cancers 2021, 13, 4484. [CrossRef]

16. Sonntag, R.; Giebeler, N.; Nevzorova, Y.A.; Bangen, J.M.; Fahrenkamp, D.; Lambertz, D.; Haas, U.; Hu, W.; Gassler, N.; Cubero, F.J.; et al. Cyclin e1 and cyclin-dependent kinase 2 are critical for initiation, but not for progression of hepatocellular carcinoma. Proc. Natl. Acad. Sci. USA 2018, 115, 9282-9287. [CrossRef]

17. Decensi, A.; Puntoni, M.; Goodwin, P.; Cazzaniga, M.; Gennari, A.; Bonanni, B.; Gandini, S. Metformin and cancer risk in diabetic patients: A systematic review and meta-analysis. Cancer Prev. Res. 2010, 3, 1451-1461. [CrossRef]

18. Zhang, Z.J.; Zheng, Z.J.; Shi, R.; Su, Q.; Jiang, Q.; Kip, K.E. Metformin for liver cancer prevention in patients with type 2 diabetes: A systematic review and meta-analysis. J. Clin. Endocrinol. Metab. 2012, 97, 2347-2353. [CrossRef]

19. Li, Y.; Casey, S.C.; Felsher, D.W. Inactivation of myc reverses tumorigenesis. J. Intern. Med. 2014, 276, 52-60. [CrossRef]

20. Poon, T.C.; Wong, N.; Lai, P.B.; Rattray, M.; Johnson, P.J.; Sung, J.J. A tumor progression model for hepatocellular carcinoma: Bioinformatic analysis of genomic data. Gastroenterology 2006, 131, 1262-1270. [CrossRef]

21. Sandgren, E.P.; Quaife, C.J.; Pinkert, C.A.; Palmiter, R.D.; Brinster, R.L. Oncogene-induced liver neoplasia in transgenic mice. Oncogene 1989, 4, 715-724.

22. Lee, J.S.; Chu, I.S.; Mikaelyan, A.; Calvisi, D.F.; Heo, J.; Reddy, J.K.; Thorgeirsson, S.S. Application of comparative functional genomics to identify best-fit mouse models to study human cancer. Nat. Genet. 2004, 36, 1306-1311. [CrossRef]

23. Dong, Y.; Tu, R.; Liu, H.; Qing, G. Regulation of cancer cell metabolism: Oncogenic myc in the driver's seat. Signal. Transduct. Target. Ther. 2020, 5, 124. [CrossRef]

24. Dang, C.V. Myc on the path to cancer. Cell 2012, 149, 22-35. [CrossRef]

25. Sunami, Y. Nash, fibrosis and hepatocellular carcinoma: Lipid synthesis and glutamine/acetate signaling. Int. J. Mol. Sci. 2020, 21, 6799. [CrossRef] 
26. Lai, K.K.Y.; Kweon, S.M.; Chi, F.; Hwang, E.; Kabe, Y.; Higashiyama, R.; Qin, L.; Yan, R.; Wu, R.P.; Lai, K.; et al. Stearoyl-coa desaturase promotes liver fibrosis and tumor development in mice via a wnt positive-signaling loop by stabilization of low-density lipoprotein-receptor-related proteins 5 and 6. Gastroenterology 2017, 152, 1477-1491. [CrossRef]

27. Reddy, J.K.; Rao, M.S. Lipid metabolism and liver inflammation. Ii. Fatty liver disease and fatty acid oxidation. Am. J. Physiol. Gastrointest. Liver Physiol. 2006, 290, G852-G858. [CrossRef]

28. Alves-Bezerra, M.; Cohen, D.E. Triglyceride metabolism in the liver. Compr. Physiol. 2017, 8, 1-8.

29. Bigornia, S.J.; Farb, M.G.; Mott, M.M.; Hess, D.T.; Carmine, B.; Fiscale, A.; Joseph, L.; Apovian, C.M.; Gokce, N. Relation of depot-specific adipose inflammation to insulin resistance in human obesity. Nutr. Diabetes 2012, 2, e30. [CrossRef] [PubMed]

30. Eckel, R.H.; Kahn, S.E.; Ferrannini, E.; Goldfine, A.B.; Nathan, D.M.; Schwartz, M.W.; Smith, R.J.; Smith, S.R. Obesity and type 2 diabetes: What can be unified and what needs to be individualized? J. Clin. Endocrinol. Metab. 2011, 96, 1654-1663. [CrossRef]

31. Lu, Y.; Zhang, L.; Zhu, R.; Zhou, H.; Fan, H.; Wang, Q. Pfkfb3, a key glucose metabolic enzyme regulated by pathogen recognition receptor tlr4 in liver cells. Ther. Adv. Endocrinol. Metab. 2020, 11, 2042018820923474. [CrossRef]

32. Gao, P.; Tchernyshyov, I.; Chang, T.C.; Lee, Y.S.; Kita, K.; Ochi, T.; Zeller, K.I.; De Marzo, A.M.; Van Eyk, J.E.; Mendell, J.T.; et al. $\mathrm{C}$-myc suppression of mir-23a/b enhances mitochondrial glutaminase expression and glutamine metabolism. Nature 2009, 458, 762-765. [CrossRef]

33. Du, K.; Chitneni, S.K.; Suzuki, A.; Wang, Y.; Henao, R.; Hyun, J.; Premont, R.T.; Naggie, S.; Moylan, C.A.; Bashir, M.R.; et al. Increased glutaminolysis marks active scarring in nonalcoholic steatohepatitis progression. Cell. Mol. Gastroenterol. Hepatol. 2020, 10, 1-21. [CrossRef]

34. Simon, J.; Nunez-Garcia, M.; Fernandez-Tussy, P.; Barbier-Torres, L.; Fernandez-Ramos, D.; Gomez-Santos, B.; Buque, X.; Lopitz-Otsoa, F.; Goikoetxea-Usandizaga, N.; Serrano-Macia, M.; et al. Targeting hepatic glutaminase 1 ameliorates non-alcoholic steatohepatitis by restoring very-low-density lipoprotein triglyceride assembly. Cell Metab. 2020, 31, 605-622. [CrossRef] [PubMed]

35. Ma, C.; Kesarwala, A.H.; Eggert, T.; Medina-Echeverz, J.; Kleiner, D.E.; Jin, P.; Stroncek, D.F.; Terabe, M.; Kapoor, V.; ElGindi, M.; et al. Nafld causes selective cd4(+) t lymphocyte loss and promotes hepatocarcinogenesis. Nature 2016, 531, 253-257. [CrossRef] [PubMed]

36. Kasznicki, J.; Sliwinska, A.; Drzewoski, J. Metformin in cancer prevention and therapy. Ann. Transl. Med. 2014, 2, 57. [CrossRef] [PubMed]

37. Akinyeke, T.; Matsumura, S.; Wang, X.; Wu, Y.; Schalfer, E.D.; Saxena, A.; Yan, W.; Logan, S.K.; Li, X. Metformin targets c-myc oncogene to prevent prostate cancer. Carcinogenesis 2013, 34, 2823-2832. [CrossRef]

38. Shen, P.; Reineke, L.C.; Knutsen, E.; Chen, M.; Pichler, M.; Ling, H.; Calin, G.A. Metformin blocks myc protein synthesis in colorectal cancer via mtor-4ebp-eif4e and mnk1-eif4g-eif4e signaling. Mol. Oncol. 2018, 12, 1856-1870. [CrossRef] [PubMed]

39. Gouw, A.M.; Margulis, K.; Liu, N.S.; Raman, S.J.; Mancuso, A.; Toal, G.G.; Tong, L.; Mosley, A.; Hsieh, A.L.; Sullivan, D.K.; et al. The MYC Oncogene Cooperates with Sterol-Regulated Element-Binding Protein to Regulate Lipogenesis Essential for Neoplastic Growth. Cell Metab. 2019, 30, 556-572. [CrossRef]

40. Yan, J.B.; Lai, C.C.; Jhu, J.W.; Gongol, B.; Marin, T.L.; Lin, S.C.; Chiu, H.Y.; Yen, C.J.; Wang, L.Y.; Peng, I.C. Insulin and Metformin Control Cell Proliferation by Regulating TDG-Mediated DNA Demethylation in Liver and Breast Cancer Cells. Mol. Ther. Oncolytics 2020, 18, 282-294. [CrossRef]

41. Issa, D.; Alkhouri, N. Nonalcoholic fatty liver disease and hepatocellular carcinoma: New insights on presentation and natural history. Hepatobiliary Surg. Nutr. 2017, 6, 401-403. [CrossRef]

42. Piscaglia, F.; Svegliati-Baroni, G.; Barchetti, A.; Pecorelli, A.; Marinelli, S.; Tiribelli, C.; Bellentani, S.; Group, H.-N.I.S. Clinical patterns of hepatocellular carcinoma in nonalcoholic fatty liver disease: A multicenter prospective study. Hepatology 2016, 63, 827-838. [CrossRef] 